Gazi University
Journal of Science
http://dergipark.gov.tr/gujs

\title{
Reliable Iterative Methods for Solving Convective Straight and Radial Fins with Temperature-Dependent Thermal Conductivity Problems
}

\author{
AL-Zahraa ABDUL NABI ${ }^{1}$ (i) , Majeed AL-JAWARY ${ }^{1, *}$ \\ ${ }^{I}$ University of Baghdad, College of Education for Pure Science (Ibn AL-Haitham), Department of Mathematics, Baghdad, Iraq
}

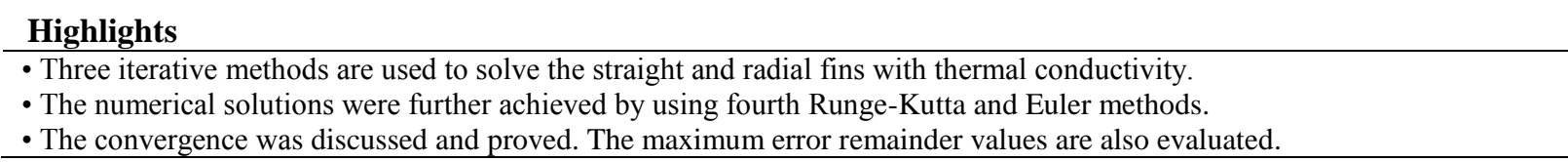

\section{Article Info}

Received: $02 / 06 / 2018$ Accepted: $13 / 11 / 2018$

\section{Keywords}

Thermal conductivity Daftardar-Jafari method Temimi-Ansari method Banach contraction method

Fins problem

\begin{abstract}
In our article, three iterative methods are performed to solve the nonlinear differential equations that represent the straight and radial fins affected by thermal conductivity. The iterative methods are the Daftardar-Jafari method namely (DJM), Temimi-Ansari method namely (TAM) and Banach contraction method namely (BCM) to get the approximate solutions. For comparison purposes, the numerical solutions were further achieved by using the fourth Runge-Kutta (RK4) method, Euler method and previous analytical methods that available in the literature. Moreover, the convergence of the proposed methods was discussed and proved. In addition, the maximum error remainder values are also evaluated which indicates that the proposed methods are efficient and reliable. Our computational works have been done by using the computer algebra system MATHEMATICA ${ }^{\circledR} 10$ to evaluate the terms in the iterative processes.
\end{abstract}

\section{INTRODUCTION}

Many phenomena in physics and engineering can be modeled by the non-linear differential equations. For example, the fin surfaces are used for developing the transfer of the heat between the base of the surface and the surrounding medium [1]. The surface area for the heat transference can be increased by thermal fins made by high conductivity materials at the base surface. Besides, the thermal conductivity for the fin materials should be high to dominate the temperature of the surface base difference to the tip of the fin. The thermal conductivity can be transfer the heat from the origin to the surface of the fin [2]. The thermal conductivity and the heat transfer coefficients are one of the key features of this is the effect on thermal conductivity and efficiency by fins [3].

There are different techniques for promoting heat transfer such as increasing the area of heat transfer coefficient or heat transfer surface. When thermal conductivity based on temperature is of great importance, this large temperature gradient will lead to an extra transfer of energy. The dependent temperature on thermal conductivity is represented by the nonlinear differential equations [4,5]. Nonlinear fin problems were of great importance to scientists and engineers because of their industrial importance and benefits in various fields of science and technology [6]. Several problems of the dimensional radioactive fins were studied and solved by the decomposition method by [7]. Recently, the equations of such problems can be transformed to a system of nonlinear differential equations and resolved as using the optimal homotopy analysis method (OHAM) [8]. Also, it can be treated and solved by the application of the Sumudu or the Fourier sine transformations [9]. Several authors have applied this kind of transformed homotopy methods in order to solve these kinds of problems, see [10-15]. 
Many applications of the fluids in sciences or engineering are made such as: the developments of the fluid models where the robustness and accuracy have been checked and evaluated with six major two-phase flow benchmark problems [16]. These problems involve two linear advection problems, the problem of oscillation for the liquid column, the problem of a ransom water faucet, the reversed water faucet problem, and the problem of the two-phase shock tube where all of them are fundamental in the nuclear engineering T-H field. Several investigations have been introduced into a numerical accuracy for the estimation of the shallow water flow based on the ensemble Kalman filter by the investigation of the Streamline-Upwind Petrov-Galerkin (SUPG), finite element method (FEM) [17]. An experimental data and results for a direct numerical simulation for the flow have been developed in a constant-cross-section tube in a passage of a shock wave through a three-layer gas system are discussed in [18]. A numerical investigation is presented on the electroosmotic flow (EOF) and solute mixing in a microchannel when considering both of the geometric modulation and surface potential heterogeneity for a wall of the channel [19].

Bartas and Sellers tested the thermal rejection system made from parallel pipes to web panels side by side [20]. Straight and radial fins have been analyzed by Coskun and Atay with thermal conductivity based on temperature using the variational iteration method (VIM) [21]. Cuce and Cuce examined the temperature and effectiveness of convection fins with the thermal conductivity based on temperature by the implementation of the homotopy perturbation scheme [22]. In addition, Chiu and Chen verified the thermal convection fins of the radiation by Adomian's decomposition method (ADM) [23,24]. Tabet et al. have applied the Padé approximations with the ADM to solve the convective straight fins in solar air collector [25]. Patra and Ray analyzed the fins of the radiological load using the homotopy perturbation Sumudu transform approach [26]. Wilkins presented the optimal ratio at the starting point of zero for the trigonometric fins radiating into space [27]. Radiator applications were studied by Cockfield as a structural part in the application of the spacecraft [28].

In this paper, three iterative methods will be used to solve the straight and radial fins with a thermal conductivity for obtain new approximate solutions. The first one is presented in 2006 by Daftardar-Gejji and Jafari namely (DJM) [29], the second iterative method is suggested in 2011 by the Temimi-Ansari method namely (TAM) [30], the third iterative method is the Banach contraction method namely (BCM), it has been presented by Varsha and Sachin Bhalekar in 2009 [31]. Moreover, for comparison purposes the governing nonlinear problems are also solved using analytic methods available in literature such as VIM and ADM, also two numerical methods are implemented.

This paper is arranged as the following: in section 2, the mathematical formulations of radial and straight thermal fins are given. Section 3 introduces the basic concepts for the three used iterative methods. Section 4 provides a solution to the problem of straight fins using the suggested methods. Section 5 provides a solution to the problem of radial fins using the suggested methods. In section 6 , the convergence of the used methods is presented. The numerical simulations and error analyses of the approximate solutions are shown in section 7 . The conclusion will be given in section 8 .

\section{THE MATHEMATICAL FORMING OF THE GOVERNING EQUATIONS}

In this part, the formulation of the straight and radiation fin problems will be presented.

\subsection{Straight Fin Problem}

Let consider a straight fin relies on $k(T)$ the temperature with heat transfer coefficient or the thermal conductivity, $S$ is some random constant area (cross-sectional) $L$ is the fin length and $p$ represents the perimeter, $T_{a}$ and $T_{b}$ are the ambient and base temperatures, in a respective way, and $h$ represents the heat transfer coefficient, see Figure 1 [32]. The 1D equation of energy balance is presented in this form $[33,34]$. 
$S \frac{d}{d x}\left(k(T) \frac{d T}{d x}\right)-p h\left(T-T_{a}\right)=0$

where $T$ is the distribution of the heat on the fin. However, we assume that the material of the fin is a linearly depend on a temperature, i.e.

$k(T)=k_{a}\left(1+\gamma\left(T-T_{a}\right)\right)$

where $k_{a}$ represents the heat conductivity at $T_{a}$ and $\gamma$ is a parameter without dimensions which describes the variance in the temperature conductivity.

$u=\frac{T-T_{a}}{T_{b}-T_{a}}, x=\frac{y}{b}, \beta=\gamma\left(T_{b}-T_{a}\right), \mu^{2}=\frac{h p L^{2}}{k_{a} S} u$

The following nonlinear equation describes the straight energy balance of fins extending to the surface under the influence, where temperature-dependent thermal conductivity is acquired [35].

$u^{\prime \prime}(x)+\beta u(x) u^{\prime \prime}(x)+\beta\left(u^{\prime}(x)\right)^{2}-\mu^{2} u(x)=0$,

with the following boundary conditions

$u^{\prime}(0)=0, \quad u(1)=1$, and $u(0)=a$.

where $u$ is an unknown function which represents the temperature without dimensions, $\mu$ thermal fin parameter, $\beta$ parameter that describes the thermal conductivity, $a$ will be evaluated later [36]. It is difficult to solve Equation (4) analytically and therefore the exact solution cannot be obtained. Hence, the approximate and numerical methods will be used.

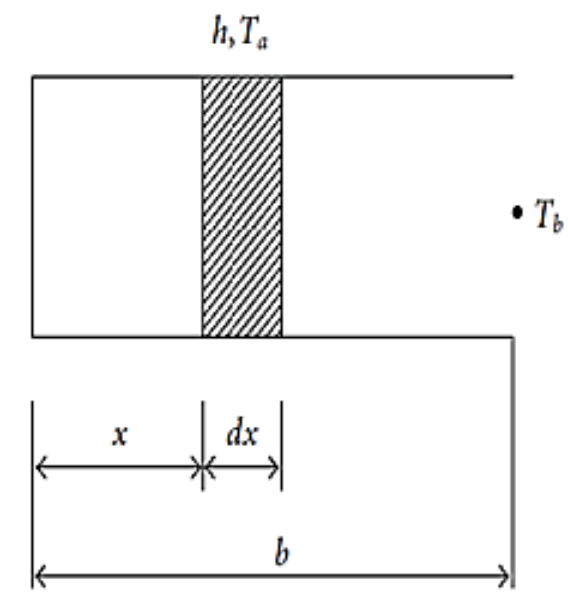

Figure 1. Geometry of the straight fin [32] 


\subsection{Radial Fin Problem}

Let us recognize the heat pipe/fin space radiator as clarified in Figure 2 [9]. As the temperature is low, the fin surfaces radiate into outer space [37]. The fin has the heat conductivity $k(T)$ that is depending on temperature linearly, $w$ is the fin diffuse with a gray balloon emission, $T_{b}$ is the core temperature, and temperature of the fixed fin tube surface [38]. The equation of the energy balance for the fin

$2 S \frac{d}{d x}\left(k(T) \frac{d T}{d x}\right)-2 w \sigma T^{4}=0$,

where the $\sigma$ represents the constant of Stefan-Boltzmann. The heat conductivity of the fin materials is considered to be a linearly depend on a temperature [39, 40].

$k(T)=k_{b}\left(1+\gamma\left(T-T_{b}\right)\right)$,

where $k_{a}$ reperesents the thermal conductivity for the fin when the core temperature, $\lambda$ is the slope of the curve between the heat conductivity and the temperature. In order to calculate a solution for Equation (6); let use these variables without dimensions.

$u=\frac{T}{T_{b}}, x=\frac{y}{b}, \beta=\gamma T_{b}, \mu=\frac{w \sigma b^{2} T_{b}^{3}}{k_{b} S}$,

Formulation of fin problem $[41,42]$ reduce to this equation

$u^{\prime \prime}(x)+\beta u(x) u^{\prime \prime}(x)+\beta\left(u^{\prime}(x)\right)^{2}-\mu(u(x))^{4}=0$,

with the following boundary conditions:

$u^{\prime}(0)=0, \quad u(1)=1$, and $u(0)=a$.

$a$ will be evaluated later. Equations (9) and (10) cannot be solved analytically and hence the approximate or numerical solutions will be obtained.

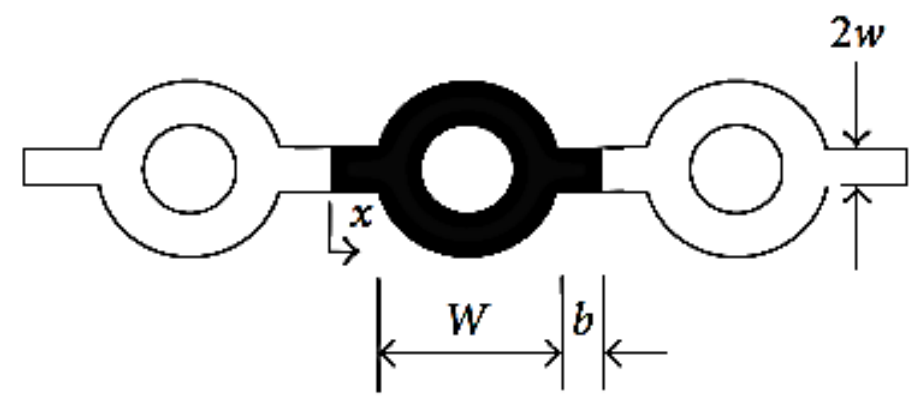

Figure 2. Schematic display of radial fin problem [9]

\section{THE MATHEMATICAL FORMULATION OF THE GOVERNING EQUATIONS}

The fundamental concepts for the three proposed techniques will be introduced in the following subsections. 


\subsection{The Basic Proceedings of the DJM}

Let us suppose the next form for a functional equation [43-46]

$u(x)=L(u)+N(u)+f$,

where $f$ is a specified function, $L$ and $N$ represent the linear and nonlinear operators, and $u$ is our goal which is an unknown function and it can be decomposed as:

$u(x)=\sum_{i=0}^{\infty} u_{i}$.

Therefore, define [47]

$G_{0}=N\left(u_{0}\right)$

$G_{k}=N\left(\sum_{i=0}^{k} u_{j}\right)-N\left(\sum_{i=0}^{k-1} u_{i}\right), k \geq 1$

So, $N(u)$ will be analyzed as follows:

$$
\begin{aligned}
N\left(\sum_{i=0}^{\infty} u_{i}\right)= & \underbrace{N\left(u_{0}\right)}_{\mathrm{G}_{0}}+\underbrace{N\left(u_{0}+u_{1}\right)-N\left(u_{0}\right)}_{\mathrm{G}_{1}}+\underbrace{N\left(u_{0}+u_{1}+u_{2}\right)-N\left(u_{0}+u_{1}\right)}_{\mathrm{G}_{2}} \\
& +\underbrace{N\left(u_{0}+u_{1}+u_{2}+u_{3}\right)-N\left(u_{0}+u_{1}+u_{2}\right)}_{\mathrm{G}_{3}}+\ldots
\end{aligned}
$$

Also, this relation will be expressed by:

$u_{0}=f$,

$u_{1}=L\left(u_{0}\right)+G_{0}$,

$u_{2}=L\left(u_{0}\right)+G_{1}$,

$\vdots$

$u_{k+1}=G_{k}+L\left(u_{k}\right)$.

As $L$ is the linear operator where $\sum_{i=0}^{k} L\left(u_{i}\right)=L\left(\sum_{i=0}^{k} u_{i}\right)$, hence

$$
\sum_{i=0}^{k+1} u_{i}=\left(\sum_{i=0}^{k} L\left(u_{i}\right)\right)+N\left(\sum_{i=0}^{k} u_{i}\right)=L\left(\sum_{i=0}^{k} u_{i}\right)+N\left(\sum_{i=0}^{k} u_{i}\right),
$$

and

$$
\sum_{i=0}^{\infty} u_{i}=f+L\left(\sum_{i=0}^{\infty} u_{i}\right)+N\left(\sum_{i=0}^{\infty} u_{i}\right)
$$

The $k$ th-term approximate solution in Equations (18) and (19) is expressed by $u=u_{0}+u_{1}+\ldots+u_{k-1}$. More details can be found in [48]. 


\subsection{The Basic Proceedings of the TAM}

Let us start with this general form of an ODE [30, 49]

$L(u(x))+N(u(x))+g(x)=0$,

with the boundary conditions

$B\left(u, \frac{d u}{d x}\right)=0$,

where $L$ is the linear operator and $N$ is the non-linear operator. The boundary operator here is $B$, and $g(x)$ is the given function. This method can be implemented as follows, assuming that $u_{0}(x)$ is the initial approximation by solving the initial problem

$L\left(u_{0}(x)\right)+g(x)=0$ and $B\left(u_{0}, \frac{d u_{0}}{d x}\right)=0$,

In order to generate the next solution, let solve the presented equation

$L\left(u_{1}(x)\right)+g(x)+N\left(u_{0}(x)\right)=0$ and $B\left(u_{1}, \frac{d u_{1}}{d x}\right)=0$.

Thus, we get a simple iterative stride that can be used to solve a set of problems

$L\left(u_{k+1}(x)\right)+g(x)+N\left(u_{k}(x)\right)=0$ and $B\left(u_{k+1}, \frac{d u_{k+1}}{d x}\right)=0$.

Note that each $u_{i}(x)$ is an approximated solution to the equation (24). The whole solution for the problem can be given by $[50,51]$

$u=\lim _{n \rightarrow \infty} u_{n}$.

\subsection{The Basic Proceedings of the $\mathrm{BCM}$}

Let us suppose the nonlinear functional equation $[52,53]$

$u(x)=f(x)+N(u(x))$,

where $u(x)$ is the unknown goal, $f(x)$ represents a known function and $N$ is the nonlinear operator for the functional Equation (26). Now we will present some consecutive approximations as:

$u_{0}=f$,

$u_{1}=u_{0}+N\left(u_{0}\right)$,

$u_{2}=u_{0}+N\left(u_{1}\right)$,

$u_{3}=u_{0}+N\left(u_{2}\right)$, 
$u_{k}=u_{0}+N\left(u_{k-1}\right), k=1,2, \ldots$

The BCM depends on the principle of contraction which we will address later. The obtained solution for Equation (28) can be presented as:

$u=\lim _{k \rightarrow \infty} u_{k}$.

\section{THE MATHEMATICAL FORMATION OF THE GOVERNING EQUATIONS}

This section shows us the straight fins will be solved by using the iterative techniques: DJM, TAM, and BCM.

\subsection{Solving the Straight Fin Problem by the DJM}

To solve the straight fin formed in Equations (4) and (5) by the DJM. The following integrated form will be obtained as described in the following steps:

$u^{\prime \prime}(x)+\beta u(x) u^{\prime \prime}(x)+\beta\left(u^{\prime}(x)\right)^{2}-\mu^{2} u(x)=0$,

rewrite Equation (30) as follows:

$u^{\prime \prime}(x)=N(u(x))$

where $N(u(x))=-\beta u(x) u^{\prime \prime}(x)-\beta\left(u^{\prime}(x)\right)^{2}+\mu^{2} u(x)$,

integrating Equation (32) from 0 to $x$, we get:

$u^{\prime}(x)=\int_{0}^{x} \int_{0}^{x} N\left(u\left(t_{1}\right)\right) d t_{1} d t_{2}$.

Also, integrating Equation (33) from 0 to $x$, we have:

$u(x)=a+\int_{0}^{x} \int_{0}^{x} N\left(u\left(t_{1}\right)\right) d t_{1} d t_{2}$,

where $u(0)=a$. For simplicity, according to the rule of reducing multiple integrals [54], the integral form given in equation (34) will be reduced to the following Volterra integral equation:

$u(x)=a+\int_{0}^{x}(x-t) N(u(t)) d t$

So, according to the basic proceedings of the DJM, we have:

$\tilde{u_{0}}(x)=a$,

$\tilde{u_{1}}(x)=\int_{0}^{x}(x-t) N\left(\tilde{u_{0}}(t)\right) d t$,

$\tilde{u_{2}}(x)=\int_{0}^{x}(x-t) N\left(\tilde{u_{1}}(t)\right) d t-u_{1}(x)$, 
The first approximation will be

$\tilde{u_{1}}(x)=a+\frac{1}{2} a x^{2} \mu^{2}$

in general, we have

$\tilde{u}_{n+1}(x)=\int_{0}^{x}(x-t) N\left(\tilde{u}_{n}(x)\right) d t-\int_{0}^{x}(x-t) N\left(\tilde{u}_{n-1}(x)\right) d t, n \in \mathrm{N}$

therefore

$$
\begin{aligned}
& \begin{aligned}
& \tilde{u_{2}}(x)=-\frac{1}{2} a^{2} x^{2} \beta \mu^{2}+\frac{1}{24} a x^{4} \mu^{4}-\frac{1}{8} a^{2} x^{4} \beta \mu^{4}, \\
& \begin{aligned}
\tilde{u_{3}}(x)= & \frac{1}{2} a^{3} x^{2} \beta^{2} \mu^{2}+\frac{1}{12} a^{2} x^{4} \beta \mu^{4}+\frac{3}{8} a^{3} x^{4} \beta^{2} \mu^{4}+\frac{1}{720} a x^{6} \mu^{6}-\frac{1}{40} a^{2} x^{6} \beta \mu^{6} \\
& +\frac{1}{12} a^{3} x^{6} \beta^{2} \mu^{6}-\frac{1}{16} a^{4} x^{6} \beta^{3} \mu^{6}-\frac{1}{1152} a^{2} x^{8} \beta \mu^{8}+\frac{1}{192} a^{3} x^{8} \beta^{2} \mu^{8}-\frac{1}{128} a^{4} x^{8} \beta^{3} \mu^{8},
\end{aligned} \\
& \text { Then } u_{3}(x)=\tilde{u_{0}}(x)+\tilde{u_{1}}(x)+\tilde{u_{2}}(x)+\tilde{u_{3}}(x) \\
&=a+\frac{1}{2} a^{3} x^{2} \beta^{2} \mu^{2}-\frac{1}{12} a^{2} x^{4} \beta \mu^{4}+\frac{3}{8} a^{3} x^{4} \beta^{2} \mu^{4}-\frac{1}{8} a^{4} x^{4} \beta^{3} \mu^{4}+\frac{1}{720} a x^{6} \mu^{6}-\frac{1}{40} a^{2} x^{6} \beta \mu^{6} \\
& \quad+\frac{1}{12} a^{3} x^{6} \beta^{2} \mu^{6}-\frac{1}{16} a^{4} x^{6} \beta^{3} \mu^{6}-\frac{1}{1152} a^{2} x^{8} \beta \mu^{8}+\frac{1}{192} a^{3} x^{8} \beta^{2} \mu^{8}-\frac{1}{128} a^{4} x^{8} \beta^{3} \mu^{8},
\end{aligned}
\end{aligned}
$$

and so on. Continuing to reach the approximation till $n=6$, for $u_{n}(x)$, where the terms are not noted to give brevity.

\subsection{Solving the Straight Fin Problem by the TAM}

To solve the problem of the straight fin problem given in Equations $(4)$ and (5) by the TAM, we have

$L(u)=u^{\prime \prime}(x), N(u)=-\beta u(x) u^{\prime \prime}(x)-\beta\left(u^{\prime}(x)\right)^{2}+\mu^{2} u(x), g(x)=0$,

we have the following initial problem

$L\left(u_{0}(x)\right)=0$, with $u_{0}(0)=a, u_{0}{ }^{\prime}(0)=0$,

in general, it will be

$L\left(u_{n+1}(x)\right)+N\left(u_{n}(x)\right)+g(x)=0, u_{n+1}(0)=a, u_{n+1}^{\prime}(0)=0$.

Through the steps in subsection (3.2), we solve the following initial problem:

$u_{0}{ }^{\prime}(x)=0, \quad u_{0}(0)=a, u_{0}{ }^{\prime}(0)=0$,

we get, 
$u_{0}(x)=a$

In the second step, we will solve the following problem:

$u_{1}^{\prime \prime}(x)=N\left(u_{0}(x)\right), u_{1}(0)=a, u_{1}{ }^{\prime}(0)=0$,

this produces

$u_{1}(x)=a+\frac{1}{2} a x^{2} \mu^{2}$

The same step for finding $u_{2}(x), u_{3}(x)$, will be used, which means solving the following problem:

$u_{2}{ }^{\prime}(x)=N\left(u_{1}(x)\right), \quad u_{2}(0)=a, u_{2}^{\prime}(0)=0$,

$u_{3}{ }^{\prime \prime}(x)=N\left(u_{2}(x)\right), \quad u_{3}(0)=a, u_{3}^{\prime}(0)=0$,

we get,

$$
\begin{aligned}
u_{2}(x)= & a+\frac{1}{2} a x^{2} \mu^{2}-\frac{1}{2} a^{2} x^{2} \beta \mu^{2}+\frac{1}{24} a x^{4} \mu^{4}-\frac{1}{8} a^{2} x^{4} \beta \mu^{4}, \\
u_{3}(x)= & a+\frac{1}{2} a^{3} x^{2} \beta^{2} \mu^{2}-\frac{1}{12} a^{2} x^{4} \beta \mu^{4}+\frac{3}{8} a^{3} x^{4} \beta^{2} \mu^{4}-\frac{1}{8} a^{4} x^{4} \beta^{3} \mu^{4}+\frac{1}{720} a x^{6} \mu^{6}-\frac{1}{40} a^{2} x^{6} \beta \mu^{6} \\
& +\frac{1}{12} a^{3} x^{6} \beta^{2} \mu^{6}-\frac{1}{16} a^{4} x^{6} \beta^{3} \mu^{6}-\frac{1}{1152} a^{2} x^{8} \beta \mu^{8}+\frac{1}{192} a^{3} x^{8} \beta^{2} \mu^{8}-\frac{1}{128} a^{4} x^{8} \beta^{3} \mu^{8},
\end{aligned}
$$

and so on. Therefore, continue to reach the approximations at $n=6$ for $u_{n}(x)$.

\subsection{Solving the Straight Fin Problem by the BCM}

To implement the BCM to solve the straight fin problem, let us begin with the same steps of integration processes in the section (4.1) and thus we get an integrated form given in Equation (34). So according to the BCM steps given in subsection 3.3, we have:

$u^{\prime \prime}(x)+\beta u(x) u^{\prime \prime}(x)+\beta\left(u^{\prime}(x)\right)^{2}-\mu(u(x))^{4}=0$,

by rewriting the Equation (43) as:

$u^{\prime \prime}(x)=N(u(x))$

where

$N(u(x))=-\beta u(x) u^{\prime \prime}(x)-\beta\left(u^{\prime}(x)\right)^{2}+\mu(u(x))^{4}$,

therefore,

$u(x)=a+\int_{0}^{x}(x-t) N(u(t)) d t$ 
then, we have

$u_{0}(x)=a$,

$u_{1}(x)=u_{0}(x)+\int_{0}^{x}(x-t) N\left(u_{0}(t)\right) d t$.

In general, we have

$u_{n+1}(x)=u_{0}(x)+\int_{0}^{x}(x-t) N\left(u_{n}(t)\right) d t, n \geq 1$.

Therefore

$$
\begin{aligned}
u_{1}(x)= & a+\frac{1}{2} a x^{2} \mu^{2}, \\
u_{2}(x)= & a+\frac{1}{2} a x^{2} \mu^{2}-\frac{1}{2} a^{2} x^{2} \beta \mu^{2}+\frac{1}{24} a x^{4} \mu^{4}-\frac{1}{8} a^{2} x^{4} \beta \mu^{4}, \\
u_{3}(x)= & a+\frac{1}{2} a x^{2} \mu^{2}+\frac{1}{2} a^{3} x^{2} \beta^{2} \mu^{2}-\frac{1}{12} a^{2} x^{4} \beta \mu^{4}+\frac{3}{8} a^{3} x^{4} \beta^{2} \mu^{4}-\frac{1}{8} a^{4} x^{4} \beta^{3} \mu^{4}+\frac{1}{720} a x^{6} \mu^{6} \\
& -\frac{1}{40} a^{2} x^{6} \beta \mu^{6}+\frac{1}{12} a^{3} x^{6} \beta^{2} \mu^{6}-\frac{1}{16} a^{4} x^{6} \beta^{3} \mu^{6}-\frac{1}{1152} a^{2} x^{8} \beta \mu^{8}+\frac{1}{192} a^{3} x^{8} \beta^{2} \mu^{8} \\
& -\frac{1}{128} a^{4} x^{8} \beta^{3} \mu^{8},
\end{aligned}
$$

and so on, continue in order to get the approximations at $n=6$ for $u_{n}(x)$.

\section{SOLVING THE RADIAL FIN PROBLEM}

In this section, the radial fin problem will be resolved by applying the three iterative processes: DJM, $\mathrm{TAM}$, and BCM.

\subsection{Solving the Radial Fin Problem by the DJM}

Consider the problem of radial fin problem in Equations (9) and (10), the following integrated form will be obtained as described in the following steps:

$u^{\prime \prime}(x)+\beta u(x) u^{\prime \prime}(x)+\beta\left(u^{\prime}(x)\right)^{2}-\mu(u(x))^{4}=0$,

Equation (47) can be written as the following:

$u^{\prime \prime}(x)=N(u(x))$

where

$$
N(u(x))=-\beta u(x) u^{\prime \prime}(x)-\beta\left(u^{\prime}(x)\right)^{2}+\mu(u(x))^{4},
$$

also, integrating Equation (48) from 0 to $x$ twice, and by employing the boundary conditions: 
$u(x)=a+\int_{0}^{x} \int_{0}^{x} N\left(u\left(t_{1}\right)\right) d t_{1} d t_{2}$,

where $u(0)=a$, then we have

$\tilde{u}_{n+1}(x)=\int_{0}^{x}(x-t) N\left(\sum_{i=0}^{n} \tilde{u_{i}}(t)\right) d t-\int_{0}^{x}(x-t) N\left(\sum_{i=0}^{n-1} \tilde{u_{i}}(t)\right) d t$,

thus, we obtain

$\tilde{u_{0}}(x)=a$,

$\tilde{u_{1}}(x)=\frac{1}{2} a^{4} x^{2} \mu$

$\tilde{u_{2}}(x)=-\frac{1}{2} a^{5} x^{2} \beta \mu+\frac{1}{6} a^{7} x^{4} \mu^{2}-\frac{1}{8} a^{8} x^{4} \beta \mu^{2}+\frac{1}{20} a^{10} x^{6} \mu^{3}+\frac{1}{112} a^{13} x^{8} \mu^{4}+\frac{a^{16} x^{10} \mu^{5}}{1440}$.

Then $u_{2}(x)=\tilde{u_{0}}(x)+\tilde{u_{1}}(x)+\tilde{u_{2}}(x)$

$$
\begin{aligned}
= & a+\frac{1}{2} a^{4} x^{2} \mu-\frac{1}{2} a^{5} x^{2} \beta \mu+\frac{1}{6} a^{7} x^{4} \mu^{2}-\frac{1}{8} a^{8} x^{4} \beta \mu^{2}+\frac{1}{20} a^{10} x^{6} \mu^{3}+\frac{1}{112} a^{13} x^{8} \mu^{4} \\
& +\frac{1}{1440} a^{16} x^{10} \mu^{5},
\end{aligned}
$$

and so on, continue to obtain the order at $n=4$ for $u_{n}(x)$.

\subsection{Solving the Radial Fin Problem by the TAM}

To solve the problem of the radial fin problem given in Equations (9) and (10) by the TAM, we begin by solving the following initial problem:

$u_{0}{ }^{\prime \prime}(x)=0, \quad u_{0}(0)=a, u_{0}{ }^{\prime}(0)=0$,

we get,

$u_{0}(x)=a$

In the second step, we solve the following problem:

$u_{1}{ }^{\prime}(x)=N\left(u_{0}(x)\right), \quad u_{1}(0)=a, u_{1}{ }^{\prime}(0)=0$,

therefore, we get

$u_{1}(x)=a+\frac{1}{2} a^{4} x^{2} \mu$

In the third step, we obtain 


$$
\begin{aligned}
u_{2}(x)= & a+\frac{1}{2} a^{4} x^{2} \mu-\frac{1}{2} a^{5} x^{2} \beta \mu+\frac{1}{6} a^{7} x^{4} \mu^{2}-\frac{1}{8} a^{8} x^{4} \beta \mu^{2}+\frac{1}{20} a^{10} x^{6} \mu^{3}+\frac{1}{112} a^{13} x^{8} \mu^{4} \\
& +\frac{1}{1440} a^{16} x^{10} \mu^{5}
\end{aligned}
$$

and so on, continue in this manner to have the approximation when $n=4$ for $u_{n}(x)$.

\subsection{Solving the Radial Fin Problem by the BCM}

To apply the BCM for the radial fins problem; let us begin with the same steps of integration processes in the subsection (5.1) and thus we get an integrated form given in Equation (50). So according to the BCM steps 3.3 , we get:

$$
\begin{aligned}
u_{0}(x) & =a, \\
u_{1}(x) & =a+\frac{1}{2} a^{4} x^{2} \mu \\
u_{2}(x) & =a+\frac{1}{2} a^{4} x^{2} \mu-\frac{1}{2} a^{5} x^{2} \beta \mu+\frac{1}{6} a^{7} x^{4} \mu^{2}-\frac{1}{8} a^{8} x^{4} \beta \mu^{2}+\frac{1}{20} a^{10} x^{6} \mu^{3}+\frac{1}{112} a^{13} x^{8} \mu^{4} \\
& +\frac{1}{1440} a^{16} x^{10} \mu^{5},
\end{aligned}
$$

and so on, continue to obtain approximations at $n=4$ for $u_{n}(x)$. It is worth to mention that the obtained approximate solutions by the three iterative methods are the same.

\section{THE CONVERGENCE OF THE PROPOSED TECHNIQUES}

In this part, let us discuss the convergence for the proposed techniques. The convergence for the DJM can be applied directly. However, to demonstrate the convergence for the TAM and BCM, we must use the following steps:

$$
\begin{aligned}
& w_{0}=u_{0}(x), \\
& w_{1}=F\left[w_{0}\right], \\
& w_{2}=F\left[w_{0}+w_{1}\right], \\
& \vdots \\
& w_{n+1}=F\left[w_{0}+w_{1}+\ldots+w_{n}\right] .
\end{aligned}
$$

where $F$ is the operator that can be defined as

$$
F\left[w_{k}\right]=S_{k}-\sum_{i=0}^{k-1} u_{i}(x), k=1,2, \ldots
$$

The term $S_{k}$ expresses the solution for one of these problems.

For the TAM:

$$
L\left(u_{k}(x)\right)+g(x)+N\left(\sum_{i=0}^{k-1} u_{i}(x)\right)=0, k=1,2, \ldots
$$

For the BCM: 
$u_{k}=u_{0}+N\left[\sum_{i=0}^{k-1} u_{i}(x)\right], k=1,2, \ldots$

The same given conditions with the used iterative method will be used. Thus, in this manner, we will have $u(x)=\lim _{n \rightarrow \infty} u_{n}(x)=\sum_{n=0}^{\infty} w_{n}$. Therefore, by using (54) and (55), one can obtain the solution by this form

$u(x)=\sum_{i=0}^{\infty} w(x)$

According to the recursive algorithm of the DJM, TAM, and BCM, We present the basic condition for conducting convergence of our methods, in these theorems [55]

Theorem 6.1. Let $F$ presented in $(55)$, be an operator from a Hilbert space $H$ to $H$. The series solution $\quad u_{n}(x)=\sum_{i=0}^{n} w_{i}(x) \quad$ is $\quad$ convergent $\quad$ if $\quad \exists 0<\gamma<1 \quad$ when $\left\|F\left[w_{0}+w_{1}+\ldots+w_{i+1}\right]\right\| \leq \gamma\left\|F\left[w_{0}+w_{1}+\ldots+w_{i}\right]\right\|$ (such that $\left.\left\|w_{i+1}\right\| \leq \gamma\left\|w_{i}\right\|\right) \forall i=0,1,2, \ldots$

This theorem derived from the Banach's fixed point theorem where it is a sufficient condition for studying the convergence for our proposed iterative techniques.

Theorem 6.2. If the series solution $u(x)=\sum_{i=0}^{\infty} w_{i}(x)$ converges, then this series represents the exact solution for the two problems given in Equations (4) and (5) and Equations (9) and (10).

Theorem 6.3. Consider the series solution $\sum_{i=0}^{\infty} w_{i}(x)$ which is presented in $(58)$ is convergent to the solution $u(x)$. If the truncated series $\sum_{i=0}^{n} w_{i}(x)$ is used as an approximation to the solution of the current problem, then the maximum error $E_{n}(x)$ can be estimated by:

$E_{n}(x) \leq \frac{1}{1-\gamma} \gamma^{n+1}\left\|w_{0}\right\|$.

Theorems 6.1 and 6.2 state that the obtained solutions from the DJM given in (17), the TAM which is given in (24), or the solution of the BCM given in (28), for the nonlinear equation (6) is convergent to the exact solution under the given conditions, $\exists 0<\gamma<1$ such that $\left\|F\left[w_{0}+w_{1}+\ldots+w_{i+1}\right]\right\| \leq \gamma\left\|F\left[w_{0}+w_{1}+\ldots+w_{i}\right]\right\| \quad\left(\right.$ that $\quad$ is $\left.\left\|w_{i+1}\right\| \leq \gamma\left\|w_{i}\right\|\right) \forall i=0,1,2, \ldots \quad$ In another meaning, for each $\operatorname{rank} i$, if the parameters are defined

$$
\rho_{i}= \begin{cases}\frac{\left\|w_{i+1}\right\|}{\left\|w_{i}\right\|}, & \left\|w_{i}\right\| \neq 0 \\ 0, & \left\|w_{i}\right\|=0\end{cases}
$$


Then the series solution $\sum_{i=0}^{\infty} w_{i}(x)$ of Equations (4) and (9) converges to the exact solution $u(x)$, when $0 \leq \rho_{i}<1, \forall i=0,1,2, \ldots$. Also, as presented in Theorem 6.3, the maximum truncation error can be estimated to be $\left\|u(x)-\sum_{i=0}^{n} w_{i}\right\| \leq \frac{1}{1-\rho} \rho^{n+1}\left\|w_{0}\right\|$, where $\rho=\max \left\{\rho_{i}, i=0,1, \ldots, n\right\}$.

\subsection{The Convergence of the DJM, TAM, and BCM for Straight Fin Problem}

In order to prove the convergence for the DJM, TAM, and BCM for the problem of the straight fin, we follow the following procedure:

when applying TAM, the $S_{k}$ represents the following problem

$u_{k}{ }^{\prime}(x)=N\left(\sum_{i=0}^{k-1} u_{i}(t)\right), \quad u_{k}(0)=a, \quad u_{k}{ }^{\prime}(0)=0, \quad k=1,2, \ldots$

Also, when applying BCM, the $S_{k}$ represents the following problem

$u_{k}=u_{0}+N\left[\sum_{i=0}^{k-1} u_{i}(x)\right], k=1,2, \ldots$

When applying the DJM, the iterations terms have been achieved in subsection 4.1. The value of $a$ is calculated by substitute the given condition $u(1)=0$ with $\beta=0.1$ and $\mu=0.2$ in $u_{6}(x)$, we get $a=0.9820752510863351$. By setting off the values of $a, \beta$ and $\mu$ in the obtained $u_{6}(x)$, one can get the approximate solution.

For assessing the convergent conditions of the obtained approximate solution we evaluate the $\rho_{i}$, we get

$$
\begin{aligned}
& \rho_{0}=\frac{\left\|w_{1}\right\|}{\left\|w_{0}\right\|}=0.9820752510863351<1 \\
& \rho_{1}=\frac{\left\|w_{2}\right\|}{\left\|w_{1}\right\|}=0.09389211652421377<1 \\
& \rho_{2}=\frac{\left\|w_{3}\right\|}{\left\|w_{2}\right\|}=0.09272590944592346<1 \\
& \rho_{3}=\frac{\left\|w_{4}\right\|}{\left\|w_{3}\right\|}=0.09250255247944411<1 \\
& \rho_{4}=\frac{\left\|w_{5}\right\|}{\left\|w_{4}\right\|}=0.09235921107655067<1 .
\end{aligned}
$$

The $\rho_{i}$ 's, for $i \geq 0$ and $0<x<1$, are less than one for the three methods (since the approximate solutions are the same). Hence, is converging according to the convergence condition. 


\subsection{The Convergence of the DJM, TAM, and BCM for Radial Fin Problem}

To determine the convergence analysis of the DJM, TAM, and BCM for the radial fin problem, we do the following:

when applying TAM, the $S_{k}$ performs the following problem

$u_{k}{ }^{\prime \prime}(x)=N\left(\sum_{i=0}^{k-1} u_{i}(t)\right), \quad u_{k}(0)=a, \quad u_{k}{ }^{\prime}(0)=0, \quad k=1,2, \ldots$

Also, when applying BCM, the $S_{k}$ represents the following problem

$u_{k}=u_{0}+N\left[\sum_{i=0}^{k-1} u_{i}(x)\right], k=1,2, \ldots$

When applying DJM, we achieved the terms given in subsection 5.1. The value of $a$ is calculated by substitute the given condition $u(1)=0$ with $\beta=0.1$ and $\mu=0.2$ in $u_{1}(x)$, we get $a=0.9263593057311732$. By adjusting the values of $a, \beta$ and $\mu$ in the obtained $u_{1}(x)$, one has the approximate solution. In order to check the convergence, we have to evaluate the $\rho_{i}$

$$
\begin{aligned}
& \rho_{0}=\frac{\left\|w_{1}\right\|}{\left\|w_{0}\right\|}=0.9263593057311732<1 \\
& \rho_{1}=\frac{\left\|w_{2}\right\|}{\left\|w_{1}\right\|}=0.6738957216238456<1 \\
& \rho_{2}=\frac{\left\|w_{3}\right\|}{\left\|w_{2}\right\|}=0.15375146157751043<1 \\
& \rho_{3}=\frac{\left\|w_{4}\right\|}{\left\|w_{3}\right\|}=0.14384893447805971<1 \\
& \rho_{4}=\frac{\left\|w_{5}\right\|}{\left\|w_{4}\right\|}=0.13621997659656196<1 .
\end{aligned}
$$

The $\rho_{i}$ 's, for $i \geq 0$ and $0<x<1$, are less than one for the three methods (since the approximate solutions are the same). Hence, is convergent according to the convergence condition

\section{THE NUMERICAL DISCUSSION AND SIMULATIONS}

\subsection{Straight Fin Problem}

The effect of the DJM, TAM, and BCM for the straight fin problem the problem was examined to calculate the accuracy of these approximate methods of solution. We can find the appropriate approximate solutions when determining $\beta, \mu$ values. We observed that the numerical results obtained using three suggested iterative methods are similar to each other. Table 1 presents different values of $\beta, \mu$ and $a$. 
Table 1. The values of $u(0)=a$ for the approximate solution the problem straight fins

\begin{tabular}{|l|l|l|}
\hline$\beta$ & $\mu$ & $\mathrm{a}_{\text {Approx. }}$ \\
\hline 0.1 & 0.2 & 0.9820752510863351 \\
\hline 0.1 & 0.4 & 0.9312301454554193 \\
\hline 0.1 & 0.6 & 0.8552043326254753 \\
\hline 0.1 & 0.8 & 0.7640232752694894 \\
\hline 0.1 & 1 & 0.6673364482492052 \\
\hline-0.1 & 0.2 & 0.9782079795428562 \\
\hline-0.1 & 0.4 & 0.9176057048560305 \\
\hline-0.1 & 0.6 & 0.8300679823446968 \\
\hline-0.1 & 0.8 & 0.7293792788472717 \\
\hline-0.1 & 1 & 0.6270320845758087 \\
\hline 0.1 & 0.2 & 0.9820752510863351 \\
\hline
\end{tabular}

Moreover, to check the accuracy the root mean square function (RMS) is calculated by

$$
\operatorname{RMS}(u)=\sqrt{\frac{\sum\left(u_{\mathrm{Approx} .}-u_{\mathrm{RK} 4}\right)^{2}}{\sum\left(u_{\mathrm{RK} 4}\right)^{2}}},
$$

where $u_{\mathrm{RK} 4}$ represents the numerical solution acquired by the Runge-Kutta 4 method (RK4) and $u_{\text {Approx. }}$ is the approximate solution we obtained using three suggested iterative methods. Since the exact solution is not available the numerical solution $\mathrm{u}_{\mathrm{RKM}}$ is used, Figures 3(a) and (b) show the RMS which can be simply seen by raising the values of $\beta$ and $\mu$ the error will be increasing.

Furthermore, the residual error function is evaluated by [45]:

$$
E R_{n}(x)=u_{n}{ }^{\prime \prime}(x)+\beta u_{n}(x) u_{n}{ }^{\prime}(x)+\beta\left(u_{n}{ }^{\prime}(x)\right)^{2}-\mu^{2} u_{n}(x)
$$

The following maximal error remainder parameter

$$
M E R_{n}=\max _{0 \leq x \leq 1}\left|E R_{n}(x)\right|
$$

For $\beta=0.1$ and $\mu=0.2$, the maximal error remainder $M E R_{n}$ values for the numerical solutions obtained by our analytic used methods, the ADM [25,34] and the VIM [9] can be plotted as in Figure 3 (c) and the convergent is clear when increasing the rank number of iterations. Moreover, Figure 3 (d) offers the results of the proposed methods that are in a good deal with the resulted solutions given by the ADM, VIM, Runge-Kutta and Euler methods. 
(a)

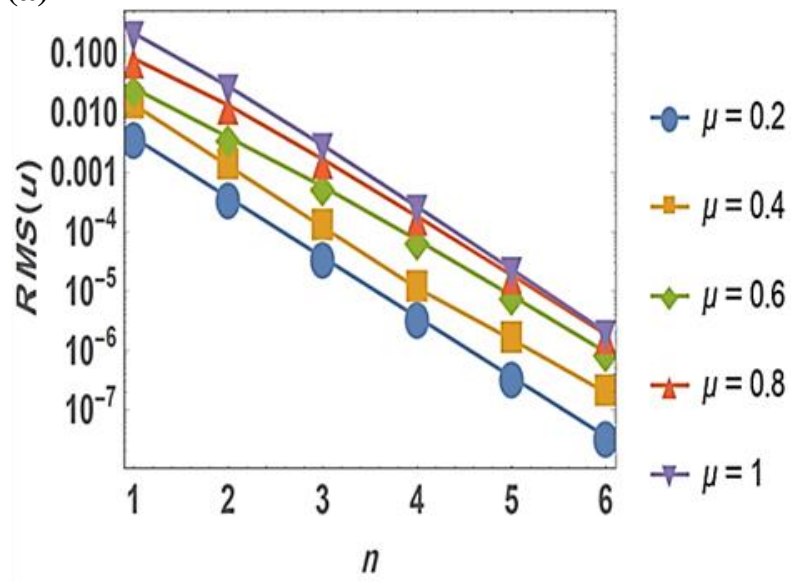

(c)

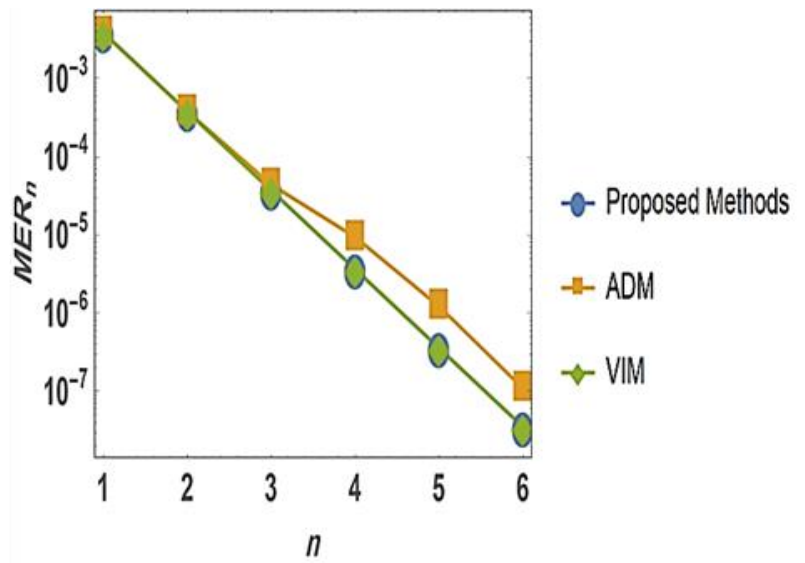

(b)

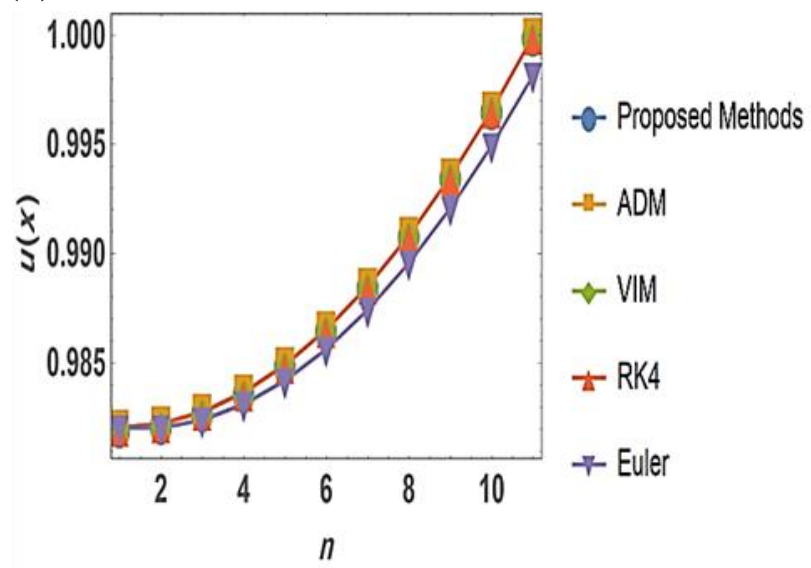

(d)

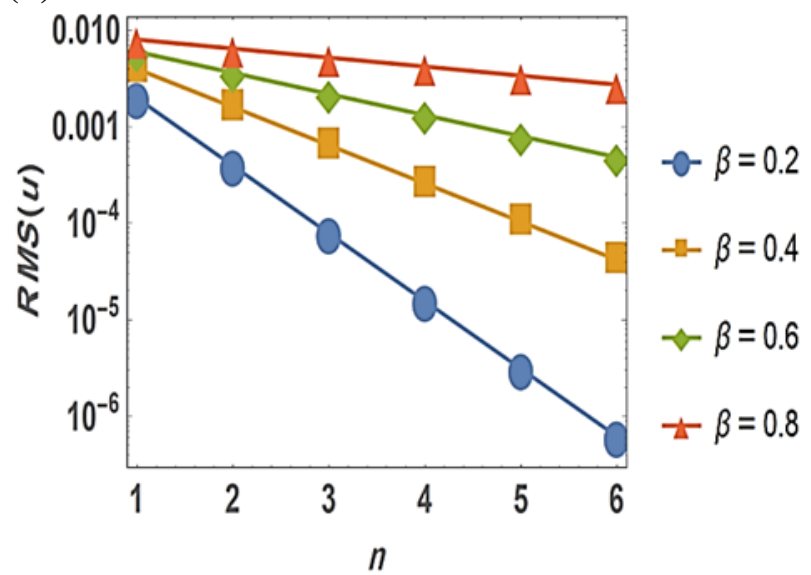

Figure 3. a) Logarithmic plots of the RMS solution the problem straight fins function for various values of $\alpha$ at $\beta=0.1 b$ ) Logarithmic plots of the RMS solution the problem straight fins function for various values of $\alpha$ at $\mu=0.1$ c) The MER $R_{n}$ the problem straight fins values obtained by proposed methods, the ADM and the VIM when $\beta=0.1$ and $\mu=0.2 d$ ) The numerical solutions obtained the problem straight fins by our proposed methods, at $\beta=0.1$ and $\mu=0.2$.d) The numerical solutions obtained the problem straight fins by our proposed methods, at $\beta=0.1$ and $\mu=0.2$

\subsection{Radial Fin Problem}

The effect of the proposed methods for solving the problem of the radial fin problem was studied. We can find the appropriate approximate solutions when determining $\beta$ and $\mu$ values. We noted that the numerical solutions achieved by the proposed methods are the same. The values of the constant missing condition $a$ are evaluated in Table 2 with several estimations of $\beta$ and $\mu$. Figures 4(a) and (b) show the root mean square function (RMS) it can be also seen when the values of $\beta$ and $\mu$ increased the error increased as well. 
Table 2. The values of $u(0)=a$ for the approximate solution by using iterations

\begin{tabular}{|l|l|l|}
\hline$\beta$ & $\mu$ & $\mathrm{a}_{\text {Approx. }}$ \\
\hline 0.1 & 0.2 & 0.9263593057311732 \\
\hline 0.1 & 0.4 & 0.8800394301309911 \\
\hline 0.1 & 0.6 & 0.8461883108282183 \\
\hline 0.1 & 0.8 & 0.8195489122835696 \\
\hline 0.1 & 1 & 0.7976231097945159 \\
\hline-0.1 & 0.2 & 0.9263593057311732 \\
\hline-0.1 & 0.4 & 0.8800394301309911 \\
\hline-0.1 & 0.6 & 0.8461883108282183 \\
\hline-0.1 & 0.8 & 0.8195489122835696 \\
\hline-0.1 & 1 & 0.7976231097945159 \\
\hline
\end{tabular}

Furthermore, the residual error function is evaluated by [45]:

$$
E R_{n}(x)=u_{n}{ }^{\prime}(x)+\beta u_{n}(x) u_{n}{ }^{\prime}(x)+\beta\left(u_{n}{ }^{\prime}(x)\right)^{2}-\mu\left(u_{n}(x)\right)^{4},
$$

the following parameter of the maximal error remainder

$$
M E R_{n}=\max _{0 \leq x \leq 1}\left|E R_{n}(x)\right|
$$

For $\beta=0.1$ and $\mu=0.2$, the $M E R_{n}$ values for the numerical results reached by our proposed methods, the ADM [25] and the VIM [9] can be plotted as in Figure 4(c) and it can be discerned obviously that the error decreases when increasing the number of iterations. Finally, Figure 4(d) shows a good agreement between the approximate solutions achieved by our proposed methods and ADM, VIM, RK4 and Euler method.

(a)

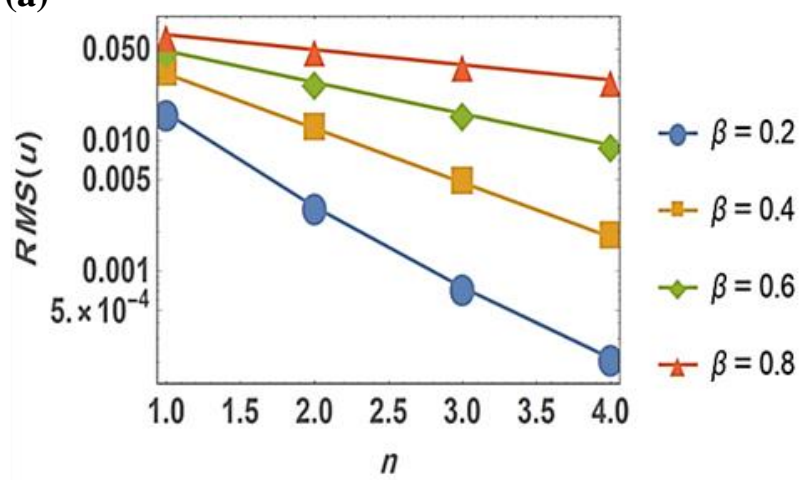

(b)

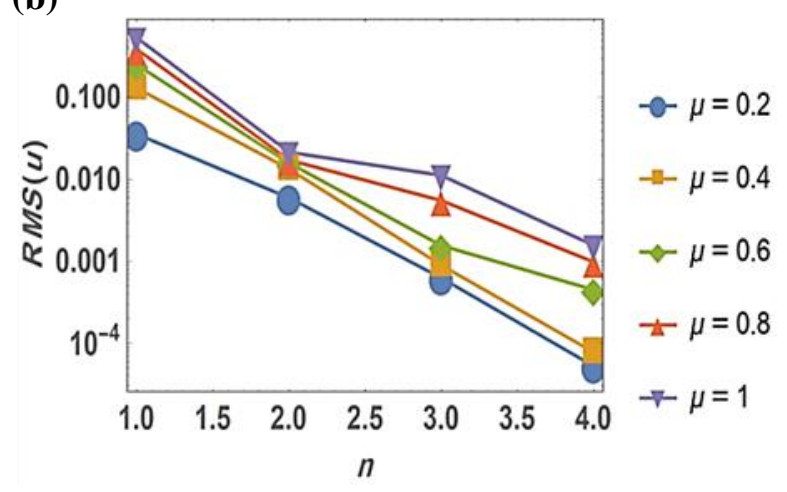


(c)

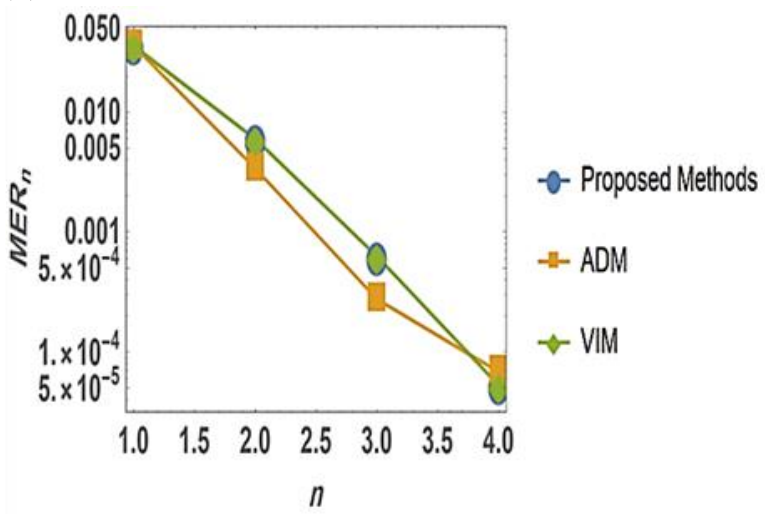

(d)

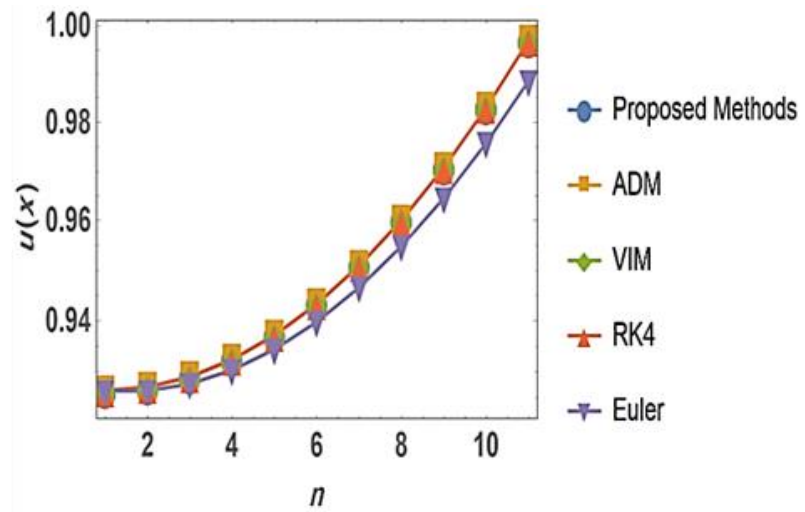

Figure 4. a) Logarithmic plots of the RMS solution the problem Radial fins function for various values of $\alpha$ at $\beta=0.1$ b) Logarithmic plots of the RMS solution the problem Radial fins function for various values of $\alpha$ at $\mu=0.1$ c) The $M_{n}$ the problem radial fins values obtained by proposed methods, the $A D M$ and the VIM when $\beta=0.1$ and $\mu=0.2$ d) The numerical solutions obtained the problem radial fins by our proposed methods, at $\beta=0.1$ and $\mu=0.2$

It should be noted that the main benefits of the obtained results that the methods have many advantages such as being derivative-free and overcoming the inconvenience arising in calculating Adomian polynomials to handle the non-linear terms in the ADM. It does not need evaluating the multiplier of Lagrange as in the VIM in which the terms of the sequence are shifted to be complex after several iterations, thus, analytical evaluation of terms becomes very difficult or impossible in the VIM. No need to create a homotopy as in the homotopy perturbation method (HPM) and solve the identical algebraic equations.

\section{CONCLUSION}

In this paper, three iterative methods namely the DJM, TAM, and BCM were used to solve straight and radial fins problems with thermal conductivity associated with temperature. Approximate solutions were obtained in a converge series. Also, when the number of iterations increases, the maximum error remainder values are reduced. Moreover, the results obtained by our proposed methods are compared with other numerical results obtained by the Runge-Kutta 4 (RK4) and Euler methods and results obtained by approximate methods accessible in the literature and good agreements have noticed. Thus, the proposed methods produce accurate, reliable and effective results.

\section{CONFLICTS OF INTEREST}

No conflict of interest was declared by the authors.

\section{REFERENCES}

[1] Kem, D. Q., Kraus, D. A., "Extended surface heat transfer”, McGraw-Hill, New York, (1972).

[2] Akindeinde, S. O., "Parker-Sochacki method for the solution of convective straight fins problem with temperature-dependent thermal conductivity", International Journal of Nonlinear Science, 20: 1-11, (2018).

[3] Mokheimer, E. M. A., "Performance of annular fins with different profiles subject to variable heat transfer coefficient”, International Journal of Heat and Mass Transfer, 45(17): 3631-3642, (2002).

[4] Ganji, D. D., Rajabi, A., "Assessment of homotopy-perturbation and perturbation methods in heat radiation equation", International Communications in Heat and Mass Transfer, 33: 391-400, (2006). 
[5] Ganji, D. D., "The application of he's homotopy perturbation method to nonlinear equations arising in heat transfer", Physics Letters, 355: 337-341, (2006).

[6] Chung, B. T. F., Zhang, B. X., "Optimization of radiating fin array including mutual irradiations between radiator elements", ASME Journal of Heat Transfer, 113(4): 814-822, (1991).

[7] Lesnic, D., Heggs, P., “A decomposition method for power-law fin-type problems”, International Communications in Heat and Mass Transfer, 31(5): 673-682, (2004).

[8] Gupta, S., Kumar, D., Singh, J., "MHD mixed convective stagnation point flow and heat transfer of an incompressible nanofluid over an inclined stretching sheet with chemical reaction and radiation", International Journal of Heat and Mass Transfer, 118: 378-387, (2018).

[9] Choudhary, A., Kumar, D., Singh, J., "A fractional model of fluid flow through porous media with mean capillary pressure", Journal of the Association of Arab Universities for Basic and Applied Sciences, 21: 59-63, (2016).

[10] Singh, J., Rashidi, M. M., Sushila., Kumar, D., "A hybrid computational approach for Jeffery-Hamel flow in non-parallel walls", Neural Computing and Applications, 10.1007/s00521-017-3198-y, (2017).

[11] Kumar, D., Singh, J., Baleanu, D., "A fractional model of convective radial fins with temperaturedependent thermal conductivity", Romanian Reports in Physics, 69(1): 103, (2017).

[12] Prakash, A., Verma, V., Kumar, D., Singh, J., "Analytic study for fractional coupled Burger's equations via Sumudu transform method", Nonlinear Engineering, 2017-0090, (2018).

[13] Kumar, D., Tchier, F., Singh, J., Baleanu, D., "An efficient computational technique for fractal vehicular traffic flow", Entropy, 20(4): 259, (2018).

[14] Singh, J., Kumar, D., Baleanu, D., Rathore, S., "An efficient numerical algorithm for the fractional Drinfeld-Sokolov-Wilson equation", Applied Mathematics and Computation, 335: 12-24, (2018).

[15] Choi, J., Kumar, D., Singh, J., Swroop, R., "Analytical techniques for system of time fractional nonlinear differential equations", Journal of the Korean Mathematical Society, 54 (4), (2017) 12091229.

[16] Chao, F., Liu, D., Shan, J., Gou, J., Wu, P., "Development of temporal and spatial high-order schemes for two-fluid seven-equation two-pressure model and its applications in two-phase flow benchmark problems", International Journal for Numerical Methods in Fluids, 88(4): 169-192, (2018).

[17] Kurahashi, T., Saito, K., "Investigation of the numerical accuracy of shallow water flow estimation based on the ensemble Kalman filter using the SUPG FEM", International Journal of Computational Fluid Dynamics, 1511048, (2018).

[18] Bodrov, E. V., Zmushko, V. V., Nevmerzhitskii, N. V., Razin, A. N., Sen'kovskii, E. D., Sotskov, E. A., "Computational and Experimental Investigation of the Development of Turbulent Mixing in a Gas Layering in Passage of a Shock Wave", Fluid Dynamics, 53(3): 385-393, (2018).

[19] Bera, S., Bhattacharyya, S., "Effects of geometric modulation and surface potential heterogeneity on electrokinetic flow and solute transport in a microchannel", Theoretical and Computational Fluid Dynamics, 32(2): 201-214, (2018). 
[20] Bartas, J. G., Sellers, W. H., "Radiation fin effectiveness", ASME Journal of Heat Transfer, 82(1): 73-75, (1960).

[21] Coskun, S. B., Atay, M. T., "Analysis of convective straight and radial fins with temperaturedependent thermal conductivity using variational iteration method with comparison with respect to finite element analysis", Mathematical Problems in Engineering, 42072, 15, (2007).

[22] Cuce, E., Cuce, P. M., "Homotopy perturbation method for temperature distribution, fin efficiency and fin effectiveness of convective straight fins with temperature-dependent thermal conductivity", Institution of Mechanical Engineers, 1-7, (2012).

[23] Chiu, C. H., Chen, C. K., "A decomposition method for solving the convective longitudinal fins with variable thermal conductivity", International Journal of Heat and Mass Transfer, 45(10): 2067-2075, (2002).

[24] Chiu, C. H., Chen, C. K., "Application of Adomian's decomposition procedure to the analysis of convective-radiative fins", ASME Journal of Heat Transfer, 125(2): 312-316, (2003).

[25] Tabet, I., Kezzar, M., Touafeka, K., Bellelb, N., Gheriebc, S., Khelifa, A., Adouane, M., “Adomian decomposition method and Padé approximation to determine fin efficiency of convective straight fins in solar air collector", International Journal of Mathematical Modelling and Computations, 5(4): 335-346, (2015).

[26] Patra, A., Ray, S. S., "Homotopy perturbation Sumudu transform method for solving convective radial fins with temperature-dependent thermal conductivity of fractional order energy balance equation”, International Journal of Heat and Mass Transfer, 76: 162-170, (2014).

[27] Wilkins Jr, J. E., "Minimizing the mass of thin radiating fins", Journal of Aerospace Science, 27(2): 145-146, (1960).

[28] Cockfield, R. D., "Structural optimization of a space radiator", Journal of Spacecraft Rockets, 5(10): 1240-1241, (1968).

[29] Daftardar-Gejji, V., Jafari, H., "An iterative method for solving nonlinear functional equations", Journal of Mathematical Analysis and Applications, 316: 753-763, (2006).

[30] Temimi, H., Ansari, A. R., "A semi-analytical iterative technique for solving nonlinear problems", Computers and Mathematics with Applications, 61: 203-210, (2011).

[31] AL-Jawary, M. A., Raham, R. K., "A semi-analytical iterative technique for solving chemistry problems", Journal of King Saud University, 29(3): 320-332, (2017).

[32] Sevilgen, G., "A numerical analysis of a convective straight fin with temperature-dependent thermal conductivity", Thermal Science, 21(2): 939-952, (2017).

[33] Adomian, G., "Solving Frontier Problems of Physics: The Decomposition Method", Kluwer Academic Publishers, Dodrecht, (1994).

[34] Arslanturk, C., "A decomposition method for fin efficiency of convective straight fins with temperature-dependent thermal conductivity", International Communications in Heat and Mass Transfer, 32: 831-841, (2005).

[35] Joneidi, A. A., Ganji, D. D., Babaelahi, M., "Differential transformation method to determine fin efficiency of convective straight fins with temperature dependent thermal conductivity", International Communications in Heat and Mass Transfer, 36(7): 757-762, (2009). 
[36] Saravanakumar, K., Ananthaswamy, V., Subha, M., Rajendran, L., "Analytical solution of nonlinear boundary value problem for fin efficiency of convective straight fins with temperature-dependent thermal conductivity", ISRN Thermodynamics, 282481, 8, (2013).

[37] Torabi, M., Yaghoobi, H., Colantoni, A., Biondi, P., Boubaker, K., "Analysis of radiative radial fin with temperature-dependent thermal conductivity using nonlinear differential transformation methods", Chinese Journal of Engineering, 470696, 12, (2013).

[38] Kumar, D., Singh, J., Baleanu, D., “A fractional model of convective radial fins with temperaturedependent thermal conductivity", Romanian Reports in Physics, 103,69, (2017).

[39] Coskun, S. B., Atay, M. T., "Analysis of convective straight and radial fins with temperaturedependent thermal conductivity using variational iteration method with comparison with respect to finite element analysis", Mathematical Problems in Engineering, 42072, 15, (2007).

[40] Domairry, G., Fazeli, M., "Homotopy analysis method to determine the fin efficiency of convective straight fins with temperature-dependent thermal conductivity", Communications in Nonlinear Science and Numerical Simulation, 14(2): 489-499, (2009).

[41] Arslanturk, C., "Correlation equations for optimum design of annular fins with temperature dependent thermal conductivity", Heat and Mass Transfer, 45(4): 519-525, (2009).

[42] Kundu, B., "Analysis of thermal performance and optimization of concentric circular fins under dehumidifying conditions", International Journal of Heat and Mass Transfer, 52(11-12): 2646-2659, (2009).

[43] Daftardar-Gejji, V., Bhalekar, S., "Solving fractional boundary value problems with Dirichlet boundary conditions using a new iterative method", Computers and Mathematics with Applications, 59(5): 1801-1809, (2010).

[44] AL-Jawary, M. A., Radhi, G. H., Ravnik, J., "Daftardar-Jafari method for solving nonlinear thin film flow problem", Arab Journal of Basic and Applied Sciences, 25(1): 20-27, (2018).

[45] AL-Jawary, M. A., "A semi-analytical iterative method for solving nonlinear thin film flow problems", Chaos, Solitons and Fractals, 99: 52-56, (2017).

[46] AL-Jawary, M. A., "An efficient iterative method for solving the Fokker-Planck equation", Results in Physics, 6: 985-991, (2016).

[47] Daftardar-Gejji, V., Jafari, H., "An iterative method for solving nonlinear functional equations", Journal of Mathematical Analysis and Applications, 316: 753-763, (2006).

[48] Bhalekar, S., Daftardar-Gejji, V., "Convergence of the new iterative method", International Journal of Differential Equations, 989065, 10, (2011).

[49] AL-Jawary, M. A., Hatif, S., "A semi-analytical iterative method for solving differential algebraic equations", Ain Shams Engineering Journal, (2017) (In press).

[50] AL-Jawary, M. A., Radhi, G. H., Ravnik, J., "Semi-analytical method for solving Fokker-Planck's equations", Journal of the Association of Arab Universities for Basic and Applied Sciences, 24: 254$262,(2017)$.

[51] AL-Jawary, M. A., Azeez, M. M., Radhi, G. H., "Analytical and numerical solutions for the nonlinear Burgers and advection-diffusion equations by using a semi-analytical iterative method", Computers and Mathematics with Applications, 76: 155-171, (2018). 
[52] Daftardar-Gejji, V., Bhalekar, S., "Solving nonlinear functional equation using Banach contraction principle", Far East Journal of Applied Mathematics, 34(3): 303-314, (2009).

[53] Al-Jawary, M. A., Adwan, M. I., Radhi, G. H., "Three iterative methods for solving second order nonlinear ODEs arising in physics”, Journal of King Saud University - Science, (2018) (In press).

[54] Wazwaz, A. M., "Linear and Nonlinear Integral Equations: Methods and Applications", Higher Education Press, Beijing and Springer-Varlag Berlin Heidelberg, (2011).

[55] Odibat, Z. M., "A study on the convergence of variational iteration method", Mathematical and Computer Modelling, 51: 1181-1192, (2010). 